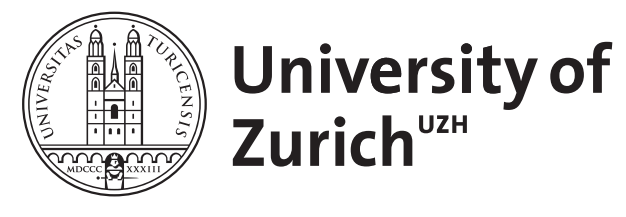

\title{
Vibrational conical intersections in the water dimer
}

\author{
Hamm, Peter ; Stock, Gerhard
}

\begin{abstract}
A recent paper (Phys. Rev. Lett. 109, 173201, 2012) has introduced the concept of vibrational conical intersections as a potential source of ultrafast vibrational relaxation, using the coupling between high-frequency $\mathrm{OH}$ modes and low-frequency intramolecular hydrogen bonding modes of malonaldehyde as an example. Here, the question is addressed whether such conical intersections may also appear for intermolecular hydrogen bonds. To that end, the water dimer is studied as a minimal model for the hydrogen bonding in liquid water. Although a significant separation of time scales between intra- and intermolecular degrees of freedom exists in (H2O)2, a standard normal mode description is found to lead to a complete break-down of the adiabatic ansatz. This is due to strong nonlinear couplings between high- and low-frequency normal modes, which in turn give rise to large overall nonadiabatic couplings. A valid adiabatic picture is obtained, on the other hand, when internal coordinates are employed. The resulting adiabatic potential energy surfaces indeed exhibit low-lying conical intersections, whose possible relevance for ultrafast relaxation and energy transfer in water is discussed.
\end{abstract}

DOI: https://doi.org/10.1080/00268976.2013.782438

Posted at the Zurich Open Repository and Archive, University of Zurich

ZORA URL: https://doi.org/10.5167/uzh-84152

Journal Article

Accepted Version

Originally published at:

Hamm, Peter; Stock, Gerhard (2013). Vibrational conical intersections in the water dimer. Molecular Physics, 111(14-15):2046-2056.

DOI: https://doi.org/10.1080/00268976.2013.782438 


\title{
Vibrational conical intersections in the water dimer
}

\author{
Peter Hamm ${ }^{1}$ and Gerhard Stock ${ }^{2}$ \\ ${ }^{1}$ Physikalisch-Chemisches Institut, Universität Zürich, \\ Winterthurerstr. 190, CH-8057 Zürich, Switzerland and \\ ${ }^{2}$ Biomolecular Dynamics, Institute of Physics, \\ Albert Ludwigs University, 79104 Freiburg, Germany
}

(Dated: March 6, 2013)

\begin{abstract}
A recent paper (Phys. Rev. Lett. 109, 173201, 2012) has introduced the concept of vibrational conical intersections as a potential source of ultrafast vibrational relaxation, using the coupling between high-frequency $\mathrm{OH}$ modes and low-frequency intramolecular hydrogen bonding modes of malonaldehyde as an example. Here, the question is addressed whether such conical intersections may also appear for intermolecular hydrogen bonds. To that end, the water dimer is studied as a minimal model for the hydrogen bonding in liquid water. Although a significant separation of time scales between intra- and intermolecular degrees of freedom exists in $\left(\mathrm{H}_{2} \mathrm{O}\right)_{2}$, a standard normal mode description is found to lead to a complete break-down of the adiabatic ansatz. This is due to strong nonlinear couplings between high- and low-frequency normal modes, which in turn give rise to large overall nonadiabatic couplings. A valid adiabatic picture is obtained, on the other hand, when internal coordinates are employed. The resulting adiabatic potential energy surfaces indeed exhibit low-lying conical intersections, whose possible relevance for ultrafast relaxation and energy transfer in water is discussed.
\end{abstract}




\section{INTRODUCTION}

Conical intersections are true crossings of two Born-Oppenheimer potential energy surfaces (PESs), which may lead to extremely fast and efficient transfer of population between the two coupled electronic states. Although conical intersections were already predicted more than 80 years ago [1], their ubiquity and utmost importance in ultrafast photochemical and photophysical processes has been recognized only much later (see Refs. [2, 3] for a timely overview). In a recent paper [4], we have shown that the concept of a conical intersection may also apply for vibrational adiabatic states. That is, while the Born-Oppenheimer ansatz is based on the adiabatic separation of time scales between the fast motion of the light electrons and the slow motion of the heavy nuclei, a similar situation also arises in the vibrational dynamics of larger molecules, e.g., when a high-frequency mode (such as a $\mathrm{OH}$ stretch vibration) is coupled to a low-frequency mode (such as a hydrogen bond mode) [5]. Adopting an adiabatic perspective, one may solve the Schrödinger equation for the high-frequency mode with the low-frequency coordinate introduced parametrically [6-11]. In direct analogy to the Born-Oppenheimer approximation for electronic states, this leads to PESs for the vibrational states of the high-frequency modes as a function of the lowfrequency coordinates.

In Ref. [4], we derived the conditions that vibrational conical intersections occur and presented quantum-dynamical model calculations to show the consequences for the vibrational dynamics and spectra. As a first simple example, we considered malonaldehyde which is a prototype molecule with a medium strong intramolecular hydrogen bond [10, 12-16]. We showed that a conical intersection exists between the first excited states of the $\mathrm{OH}$ stretch and the $\mathrm{OH}$ bend modes, respectively, which might provide an explanation for the ultrafast (a few $100 \mathrm{fs}$ ) vibrational relaxation and the complex oscillatory features found in infrared time-resolved pump-probe experiments for this type of molecular systems [17-21].

Another paradigm of ultrafast vibrational dynamics is water in its various condensed phase forms [22-29]. In isotope diluted water, where the $\mathrm{OH}$ (or OD) vibration is spectrally and spatially isolated, vibrational relaxation times in the order of 1-2 ps have been observed [30,31], which is still within the "normal" range of vibrational relaxation. In neat water, in contrast, vibrational relaxation appears to be even faster both in the liquid [32, 33] and the icy [34] state. An extremely fast initial signal on the order of $200 \mathrm{fs}$ is found, which tentatively has been attributed to vibrational energy relaxation. However, in both cases, this assignment to vibrational energy relaxation is no longer clear since the signal does not decay to zero, but into a "heated" state, which dissipates energy from the OH-excited state into intermolecular degrees of freedom. The distinction of what is called "OH excited state" and "heated" state breaks down as the adiabatic approximation breaks down. When vibrational energy relaxation occurs on timescale of a few 100 fs, which comprises only a few tens of 
oscillation periods of an $\mathrm{OH}$-stretch vibration, a perturbative treatment, which works well for most vibrations with somewhat longer relaxation times [29, 35-38], does no longer seem to be appropriate.

In the case of neat water, the situation is further complicated by the presence of energy transport along coupled (and approximately resonant) high-frequency $\mathrm{OH}$ vibrations. This kind of vibrational energy transfer is conceptually similar to excitation transport via excitonic coupling [23, 39-41]. As both processes, vibrational energy relaxation and transport, in an adiabatic perspective are viewed as nonadiabatic transitions between coupled highfrequency vibrational states $[42,43]$, the question arises if vibrational conical intersections may also be responsible for the ultrafast relaxation and energy transfer in water.

As a first step towards this question, in this work we consider the water dimer $\left(\mathrm{H}_{2} \mathrm{O}\right)_{2}$ as minimal model for the hydrogen bonding in liquid water, whose potential energy surface, structure and and vibrational spectroscopy has been studied in great detail [9, 44-52]. The hydrogen bond in the water dimer in the gas phase is relatively weak, and the IR spectra do not yet evolve the same complicated structure as stronger intramolecular hydrogen bonds [17-21]. Nevertheless, because of its obvious importance for the understanding of liquid water, whose peculiar properties have their origin in hydrogen bonding, the "water dimer serves as a sort of hydrogen atom, or prototype, of hydrogen bonding" [44].

Employing a recently published water potential of Bowman and coworkers [53-55], we determine the adiabatic PESs pertaining to the first few eigenstates of the $\mathrm{OH}$ bend and stretch vibrations of $\left(\mathrm{H}_{2} \mathrm{O}\right)_{2}$ via direct diagonalization of the six-dimensional Hamiltonian pertaining to these high-frequency vibrations [7-9]. As a standard normal-mode representation of $\left(\mathrm{H}_{2} \mathrm{O}\right)_{2}$ is found to lead to a complete break-down of the adiabatic ansatz, we adopt internal coordinates, i.e., we use normal modes of each monomer to describe the intramolecular motion of each water molecule and intermolecular coordinates to describe the relative motion of the two molecules. One and two-dimensional plots of the resulting adiabatic PESs along selected intermolecular coordinates clearly exhibit energetically low-lying conical intersections. The possible relevance of these intersections in the discussion of ultrafast relaxation and energy transfer in water is discussed. 


\section{THEORY AND METHODS}

\section{A. Adiabatic ansatz}

The adiabatic description of vibrational dynamics follows in direct analogy the standard Born-Oppenheimer approach to separate electronic and nuclear degrees of freedom. In order to introduce a separation between fast and slow degrees of freedom, we partition the vibrations into $M$ high-frequency modes $q=\left\{q_{n}\right\}$ and $N$ low-frequency modes $Q=\left\{Q_{i}\right\}$, and write the Hamiltonian as

$$
H=T_{q}+T_{Q}+V(q, Q) .
$$

Here $T_{q}$ and $T_{Q}$ denote the kinetic energy of the high- and low-frequency modes, respectively, and $V(q, Q)$ represents the electronic ground state potential energy surface. By keeping the low-frequency modes $Q_{i}$ fixed to a constant value, we first solve the Schrödinger equation for the high-frequency modes

$$
\left[T_{q}+V(q, Q)-W_{k}(Q)\right]\left|\phi_{k}\right\rangle=0
$$

which yields the vibrational adiabatic PESs $W_{k}(Q)$ and the corresponding adiabatic vibrational states $\left|\phi_{k}\right\rangle$. We now expand the total vibrational wave function as

$$
|\Phi(Q)\rangle=\sum_{k} \Psi_{k}(Q)\left|\phi_{k}\right\rangle
$$

where the dynamics of the low-frequency modes is represented by the wave functions $\Psi_{k}(Q)$. Insertion into the Schrödinger equation for the total system (1) yields the coupled-channel equations

$$
\left[T_{Q}+W_{k}(Q)-E\right] \Psi_{k}(Q)=\sum_{k^{\prime}} \Lambda_{k k^{\prime}} \Psi_{k^{\prime}}(Q),
$$

where the operator $\Lambda_{k k^{\prime}}=\left\langle\phi_{k}\left|T_{Q}\right| \phi_{k^{\prime}}\right\rangle$ accounts for the nonadiabatic coupling between the adiabatic vibrational states $\left|\phi_{k}\right\rangle$ and $\left|\phi_{k}^{\prime}\right\rangle$. In the Born-Oppenheimer approximation, one assumes that $\Lambda_{k k^{\prime}}=0$, which leads to independent low-frequency vibrational dynamics on the adiabatic PESs $W_{k}(Q)$.

\section{B. Normal-mode model}

To discuss the main features of the nonadiabatic dynamics exhibited by Eq. (4) and to introduce the concept of a vibrational conical intersection, it is instructive to employ a simple model for the vibrational system. To this end, we assume that the high-frequency and low-frequency modes are well described by dimensionless normal-mode coordinates (again 


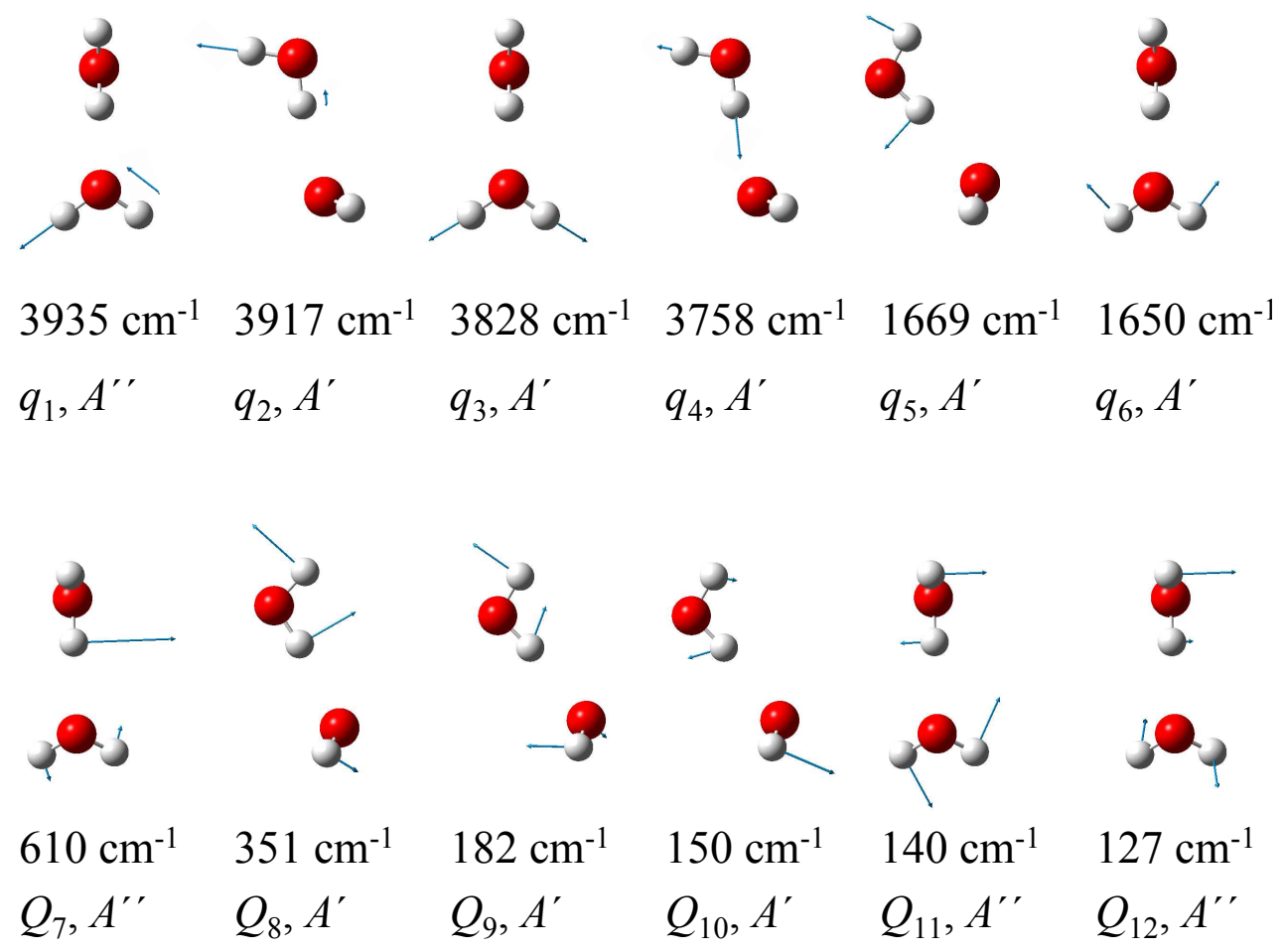

FIG. 1: Displacements, harmonic frequencies and symmetries of the normal modes of the lowest energy structure of the water dimer. Top row: intramolecular, high-frequency degrees of freedom $q_{1}$ to $q_{6}$. Bottom row: intermolecular, low-frequency degrees of freedom $Q_{7}$ to $Q_{12}$.

denoted by $\left\{q_{n}\right\}$ and $\left.\left\{Q_{i}\right\}\right)$. For the example of the water dimer, the normal modes are comprised in Fig. 1. We perform a Taylor expansion of the potential energy, which allows us to rewrite the Hamiltonian $(1)$ as $(\hbar \equiv 1)$

$$
\begin{aligned}
H & =H_{0}+H_{\mathrm{anh}}, \\
H_{0} & =\sum_{n} \frac{\omega_{n}}{2}\left(p_{n}^{2}+q_{n}^{2}\right)+\sum_{j} \frac{\Omega_{j}}{2}\left(P_{j}^{2}+Q_{j}^{2}\right),
\end{aligned}
$$

where $H_{0}$ contains the harmonic part and $H_{\text {anh }}$ all anharmonic couplings. For interpretational purposes, it is furthermore advantageous to expand the total vibrational wave function $|\Phi(Q)\rangle=\sum_{k} \Psi_{k}(Q)|k\rangle$ in terms of the zero-order harmonic basis states $|k\rangle$, with $k=\left(k_{1} \ldots k_{M}\right)$ comprising the quantum numbers of the $M$ high-frequency modes. Representation of Hamiltonian (5) in this basis yields

$$
\begin{aligned}
\mathcal{H} & =h_{0} \mathbf{1}+\sum_{k, k^{\prime}}|k\rangle h_{k k^{\prime}}\left\langle k^{\prime}\right|, \\
h_{0} & =\left\langle k\left|H_{0}\right| k^{\prime}\right\rangle=\delta_{k k^{\prime}} \varepsilon_{k}+\delta_{k k^{\prime}} \sum_{j} \frac{\Omega_{j}}{2}\left(P_{j}^{2}+Q_{j}^{2}\right), \\
h_{k k^{\prime}} & =\left\langle k\left|H_{\mathrm{anh}}\right| k^{\prime}\right\rangle,
\end{aligned}
$$


where 1 is the unit matrix and $\varepsilon_{k}=\sum_{n}\left(k_{n}+1 / 2\right) \omega_{n}\left(k_{n}=0,1,2, \ldots\right)$ denotes the harmonic energy of the high-frequency modes. Containing a diagonal kinetic energy and a nondiagonal potential energy matrix $h_{k k^{\prime}}(Q)$, Hamiltonian (7) can be regarded as a diabatic representation of the vibrational problem.

To characterize the matrix elements of Hamiltonian (7) and to explain how they give rise to vibrational conical intersections, it is helpful to restrict ourselves to the couplings

$$
H_{\mathrm{anh}}=\sum_{n, m, j} f_{n m, j} q_{n} q_{m} Q_{j}+\sum_{n, j} g_{n n, j j} q_{n}^{2} Q_{j}^{2}
$$

where $f_{n m, j}=1 / 3 ! \partial^{3} V / \partial q_{n} \partial q_{m} \partial Q_{j}$ and $g_{n m, i j}=1 / 4 ! \partial^{4} V / \partial q_{n} \partial q_{m} \partial Q_{i} \partial Q_{j}$ are the cubic and quartic coefficients of the Taylor expansion, respectively. Insertion into Eq. (7) yields

$$
\begin{aligned}
h_{k k} & =\sum_{j} \kappa_{k, j} Q_{j}+\frac{\delta \omega_{k, j}}{2} Q_{j}^{2}, \\
h_{k k^{\prime}} & =\sum_{j} \lambda_{k k^{\prime}, j} Q_{j} \quad\left(k \neq k^{\prime}\right),
\end{aligned}
$$

where nonresonant terms were neglected. The coupling constants are given by

$$
\begin{aligned}
\delta \omega_{k, j} & =\sum_{n, m} 6 g_{n n, j j}\left\langle k\left|q_{n}^{2}\right| k\right\rangle \\
\kappa_{k, j} & =\sum_{n} 3 f_{n n, j}\left\langle k\left|q_{n}^{2}\right| k\right\rangle \\
\lambda_{k k^{\prime}, j} & =\sum_{n, m} 6 f_{n m, j}\left\langle k\left|q_{n} q_{m}\right| k^{\prime}\right\rangle=\lambda_{k^{\prime} k, j}
\end{aligned}
$$

where the prefactors account for the fact that there are 3 and 6 possibilities to permute the order of the different vibrational modes in the Taylor expansion, respectively.

The first term [Eq. (11)] simply describes the frequency shift $\delta \omega_{k, j}$ of low-frequency mode $Q_{j}$ in high-frequency state $|k\rangle$, which is caused by quartic coupling. The other two couplings are only nonzero, if the corresponding matrix elements $\left\langle\Psi_{k}\left|Q_{j}\right| \Psi_{k}\right\rangle$ and $\left\langle\Psi_{k}\left|Q_{j}\right| \Psi_{k^{\prime}}\right\rangle$ do not vanish by symmetry. This means that the low-frequency mode $Q_{j}$ needs to be totally symmetric to obtain non-zero diagonal couplings $\kappa_{k, j}$. According to Eq. (9), these modes modulate the vibrational excitation energy of the high-frequency modes and are therefore referred to as "tuning coordinates". Nonzero off-diagonal couplings $\lambda_{k k^{\prime}, j}$, on the other hand, require that the direct product of the symmetry representations pertaining to $\Psi_{k}, Q_{j}$, and $\Psi_{k^{\prime}}$ includes the totally symmetric representation $\Gamma_{A}$, that is, $\Gamma_{k} \times \Gamma_{j} \times \Gamma_{k^{\prime}} \supset \Gamma_{A}[56]$. These modes couple the high-frequency modes, with the amount of the mixing depending on $Q_{j}$ (i.e., the mixing vanishes for $Q_{j}=0$ ). They are therefore referred to as "coupling coordinates". In molecules with low symmetry, vibrations may exist that simultaneously act as tuning and coupling modes. Moreover, higher-order couplings (e.g., $\propto q_{n} q_{m} Q_{j}^{2}$ ) of various symmetries may exist. 
The model defined by Eqs. (7) - (13) has several interesting features. First we notice that due to the harmonic ansatz the matrix elements of the Hamiltonian (7) are readily obtained in analytic form, which facilitates the interpretation of the vibrational interactions, e.g., in terms of tuning and coupling modes. Most interestingly, we note that the above model of vibrational coupling between high- and low-frequency modes is formally entirely equivalent to the standard linear vibronic-coupling Hamiltonian [56], which accounts for excited electronic states that interact via vibrational tuning and coupling coordinates. That is, the model may give rise to a conical intersection of the adiabatic PESs $W_{k}(Q)$, which are obtained through diagonalization of the diabatic potential-energy matrix $h_{k k^{\prime}}$. As a simple illustration, we consider the case of two coupled high-frequency vibrations with excited states $|k\rangle=|10\rangle$ and $|01\rangle$. The corresponding two adiabatic PESs are given by

$$
W_{\mp}(Q)=\bar{V}(Q) \mp \sqrt{\Delta V^{2}(Q)+V_{C}^{2}(Q)},
$$

where $\bar{V}=\left(h_{10,10}+h_{01,01}\right) / 2, \Delta V=\left(h_{10,10}-h_{01,01}\right) / 2$ and $V_{C}=h_{10,01}=h_{01,10}$. A conical intersection of these two surfaces arises for $W_{-}=W_{+}$, that is, if $\Delta V(Q)=0$ and $V_{C}(Q)=0$. In the simplest case of a single tuning and a single coupling coordinate (i.e., $N=2$ ), we obtain a crossing point, in general we have a $(N-2)$-dimensional intersection surface.

\section{Internal coordinate representation}

Being concerned with the vibrational dynamics of the water dimer, we need to describe the coupled motions of two molecules. Due to the absence of strong through-bond interactions, these molecules may perform large-amplitude motions with respect to each other. Using normal coordinates of the complete system (defined with respect to the overall potential energy minimum), these motions are only poorly described. That is, one typically obtains strong nonlinear couplings between the normal modes [57], which hamper a simple and correct picture of the vibrational motion.

As an alternative, one may invoke a set of internal coordinates of a multi-molecular system. That is, we use the normal modes of each monomer (i.e. symmetric stretch, asymmetric stretch and bend) to describe the intramolecular motion and the intermolecular coordinates defined in Fig. 2 to describe the relative motion of the molecules. This procedure again partitions the vibrations into $M$ high-frequency coordinates $x=\left\{x_{n}\right\}$ and $N$ low-frequency coordinates $X=\left\{X_{i}\right\}$. In the case of the water dimer, it yields $M=2 \times 3$ modes $\left(x_{1}, x_{2}, x_{3}\right)$ and $\left(x_{4}, x_{5}, x_{6}\right)$ to describe the intramolecular normal modes of each of the two water molecules, and $N=6$ low-frequency degrees of freedom to account for the intermolecular motion. The latter are defined by the curvilinear coordinates $X=\left(X_{7}, \ldots, X_{12}\right)$ shown in Fig. 2, i.e., the $\mathrm{O}-\mathrm{O}$ distance $R_{\mathrm{O} \ldots \mathrm{O}}$ and five rotational angles $\alpha_{y}, \alpha_{z}, \beta_{x}, \beta_{y}$, and 


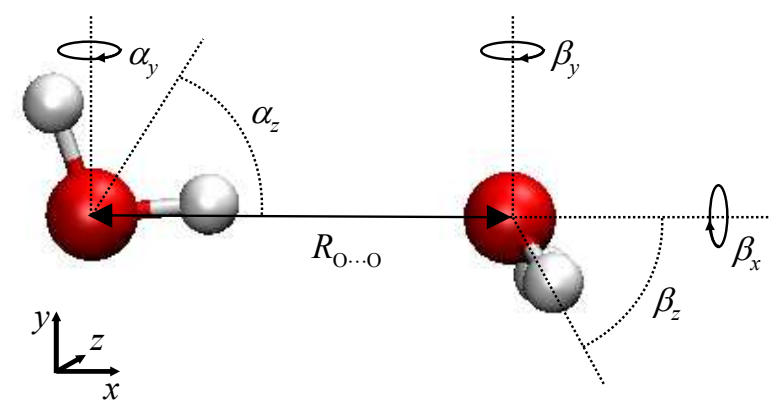

FIG. 2: Intermolecular coordinates defining the structure of the water dimer, i.e. the $\mathrm{O} \cdots \mathrm{O}$ distance $R_{\mathrm{O} \ldots \mathrm{O}}$, the rotation angles $\alpha_{y}$ and $\alpha_{z}$ of the hydrogen-bond donating water, and rotation angles $\beta_{x}, \beta_{y}$ and $\beta_{z}$ of the hydrogen-bond accepting water around axis's $x, y$, and $z$.

$\beta_{z}$. For a given geometry $X$, the associated modes $x_{n}$ are defined in a coordinate system that is fixed to the corresponding monomer. As one of the monomers rotates along one of the angles $\alpha_{y}, \alpha_{z}, \beta_{x}, \beta_{y}$, and $\beta_{z}$, the three monomer-mode vectors $\left(x_{1}, x_{2}, x_{3}\right)$ or $\left(x_{4}, x_{5}, x_{6}\right)$ of the corresponding water molecule are rotated as well (see Fig. 3c). The monomer modes therefore become a function of the rotational low-frequency degrees of freedom, $x_{n}=x_{n}(X)$. The translation of a molecule along the $\mathrm{O}-\mathrm{O}$ vector $R_{\mathrm{O} \ldots \mathrm{O}}$, in contrast, has no effect on the monomer mode vectors $x_{n}$.

In the discussion below, we wish to compare adiabatic PESs that were calculated in the internal-mode representation (using $x, X$ ) and in the normal-mode representation (using $q, Q)[58]$. To obtain a suitable transformation between the two sets of coordinates, we first calculate the normal modes in the $\left\{x_{n}\right\}$-subspace by diagonalizing the corresponding Hessian matrix, which yields the normal modes $\left\{q_{n}^{\prime}\right\}$ as linear combinations of the monomer modes

$$
q_{n}^{\prime}=\sum_{m} a_{n m} x_{m}
$$

Moreover, we introduce low-frequency normal modes $\left\{Q_{j}^{\prime}\right\}$ defined in terms of the intermolecular coordinates $\left\{X_{j}\right\}$. Since the latter are curvilinear coordinates, we need to invoke nonlinear transformation operators $\tilde{X}_{j}$, which account for the translation or rotation associated with coordinate $X_{j}$. For example for $X_{2}=\alpha_{y}, \tilde{X}_{2}$ is a rotation matrix $D_{y}\left(\alpha_{y}\right)$ around the $y$-axis with angle $\alpha_{y}$, which acts on the Cartesian coordinates $R=\left\{R_{\alpha}\right\}$ of the hydrogen-bond donating water molecule. The desired normal mode coordinates are then given by $Q_{j}^{\prime}=\tilde{Q}_{j} R$ with

$$
\tilde{Q}_{j}=\sum_{i} A_{j i} \tilde{X}_{i}
$$

In order to determine the transformation matrix $A_{i j}$, we first linearized the transformations $\tilde{X}_{i}$ for small displacements and removed the center-of-mass motion and the angular momentum from the coordinates. This yields linear vectors $Q_{j}^{\prime}$ that can be compared to $Q_{j}$. 

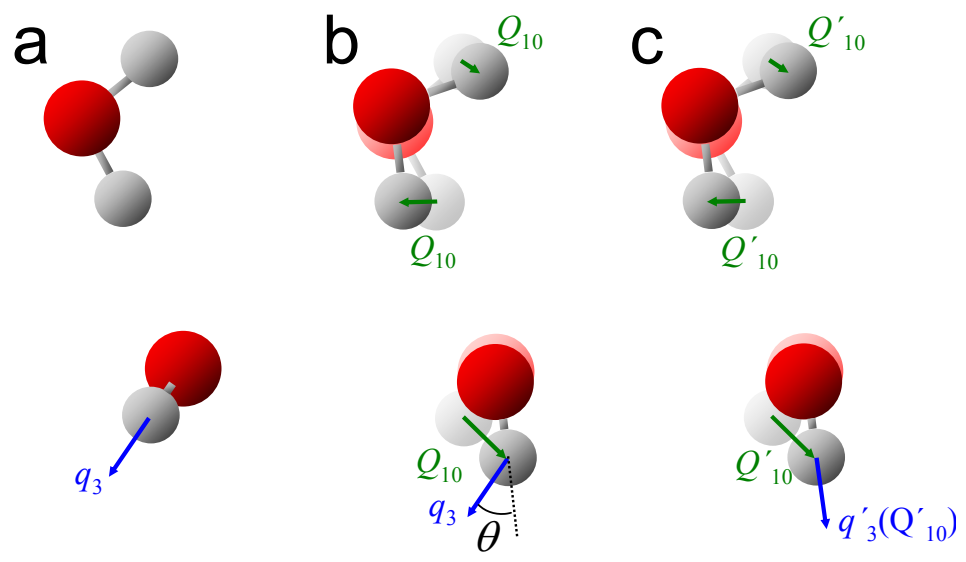

FIG. 3: (a) The symmetric stretch vibration of the hydrogen-bond accepting water (mode $q_{3}$, indicated by blue arrows) for the dimer in its minimum energy configuration. (b) In a normal-mode representation, when the molecule is displaced with respect to $Q_{10}$ (indicated by green arrows for $Q_{10}=2.0$, the minimum energy configuration is shown in the background for comparison), normal mode $q_{3}$ (in blue) no longer displaces the hydrogens parallel to the $\mathrm{OH}$ bonds, but with an angle $\theta$ out of the plane of the hydrogen-bond accepting water. (c) The same in an internal mode representation, where the high-frequency mode $q_{3}^{\prime}$ is fixed to the monomer and hence rotates as the molecule rotates.

Close to the minimum energy configuration, we find $Q_{j}^{\prime} \approx Q_{j}$ and $q_{n}^{\prime} \approx q_{n}$. This is no longer the case, when the system is displaced from its minimum energy configuration, where on the one hand we have $\left\{q_{n}\right\}=$ const., and on the other hand we have $q_{n}^{\prime}=q_{n}^{\prime}\left(Q^{\prime}\right)$, i.e., the high-frequency modes $q_{n}^{\prime}$ become a nonlinear function of the low-frequency modes. The low-frequency coordinates $\left\{Q_{i}^{\prime}\right\}$ agree with $\left\{Q_{i}\right\}$ as long as the former can be approximated by their linearized counterparts.

The effect is illustrated in Fig. 3, adopting $q_{3}$ and $Q_{10}$ as a representative example. Fig. 3a shows the displacement vector of mode $q_{3}$ for the dimer in its minimum energy configuration, while Fig. 3b shows the same high-frequency mode for the dimer displaced along $Q_{10}$. As $Q_{10}$ includes a rotation of the hydrogen-bond accepting water molecule, the normal-mode $q_{3}$ no longer displaces the proton parallel to the $\mathrm{OH}$ bond, but with an angle $\theta$ out of the plane of the hydrogen-bond accepting water. Hence, normal-mode $q_{3}$ to a certain extent rotates the water molecule back, so the high-frequency modes $q_{3}$ start to include low-frequency degrees of freedom. In contrast, in an internal coordinate representation (Fig. 3c), the mixing of low- and high-frequency degrees of freedom is avoided by construction, since we rotate the high-frequency coordinate $q_{3}^{\prime}$ as the water rotates along the low-frequency coordinate $Q_{10}^{\prime}$. 


\section{Calculation of adiabatic potential-energy surfaces}

The electronic PES of the water dimer was calculated at two levels of $a b$ initio theory. As a state-of-the-art description of the water dimer, we used a parameterized multidimensional water potential derived from aug-cc-pVTZ/CCSD(T) ab initio calculations, which was made available by Bowman and coworkers [53-55]. In this case, the potential $V(q, Q)$ was used in Schrödinger equation (2) directly. For a more qualitative discussion, we also used a Taylor expansion which directly gives the coupling terms [cf. Eq. (8)] of the simple model of nonadiabatic coupling introduced above. Harmonic frequencies and anharmonic terms up to the 4th-order were calculated with the Gaussian program package [59] on the MP2/6$311++\mathrm{g}(2 \mathrm{df}, 2 \mathrm{pd})$ level of theory. The normal modes calculated from both levels of theory were virtually the same (Fig. 1).

In the next step, we calculated the vibrational adiabatic PESs associated with the ground and lowest excited states of the high-frequency modes. In the case of the parameterized water potential of Bowman [53-55], we need to numerically solve the Schrödinger equation (2) for the high frequency modes $q_{n}$ in order to obtain the eigenstates $\phi_{k}$ and the eigenenergies (or adiabatic PESs) $W_{k}$, which both depend parametrically on the low frequency coordinates $Q_{j}$. The potential energy is given in the corresponding set of coordinates (normal or internal coordinates, respectively). In case of the internal coordinates, the intramolecular normal modes of the two monomers are used for the kinetic energy operator of the high-frequency modes (which are the only ones needed at this stage). We define a basis $\left\{\varphi_{k}\right\}$ by the product ansatz

$$
\varphi_{k}=\prod_{n=1,6} \varphi_{i, k_{n}}\left(q_{n}\right)
$$

where $k=\left(k_{1} \ldots k_{6}\right)$ comprises all quantum numbers. For a given set of fixed coordinates $\left\{Q_{j}\right\}$, the single-coordinate function $\varphi_{i, k_{n}}\left(q_{n}\right)$ is the $k_{n}$ th eigenstate of high-frequency mode $q_{n}$ with respect to the one-dimensional (1D) Schrödinger equation

$$
\left[\frac{\omega_{n}}{2} p_{n}^{2}+\mathcal{V}\left(q_{n}\right)\right] \varphi_{i, k_{n}}=\epsilon_{i, k_{n}} \varphi_{i, k_{n}}
$$

where $\mathcal{V}\left(q_{n}\right)$ denotes a 1D cut of the potential energy along $q_{n}$ with all other coordinates $q_{j \neq i}$ set to zero. To solve the 6D Schrödinger equation (2), the Hamiltonian $H_{q}=T_{q}+V(q, Q)$ was represented by the basis functions $\varphi_{k}$, where the potential energy matrix was calculated by full quadrature and the associated kinetic energy matrix according to Ref. [60]. We included all 1D functions with up to 6 excitations in total in the $\mathrm{OH}$ stretches and/or 12 excitations in the $\mathrm{OH}$ bends, thus yielding $\approx 2300$ basis functions. The Hamilton matrix was diagonalized and the resulting adiabatic states are characterized according to their largest overlap with the zero-order states $\varphi_{k}$ (which is used for the coloring in Figs. 4, 5 and 6). 

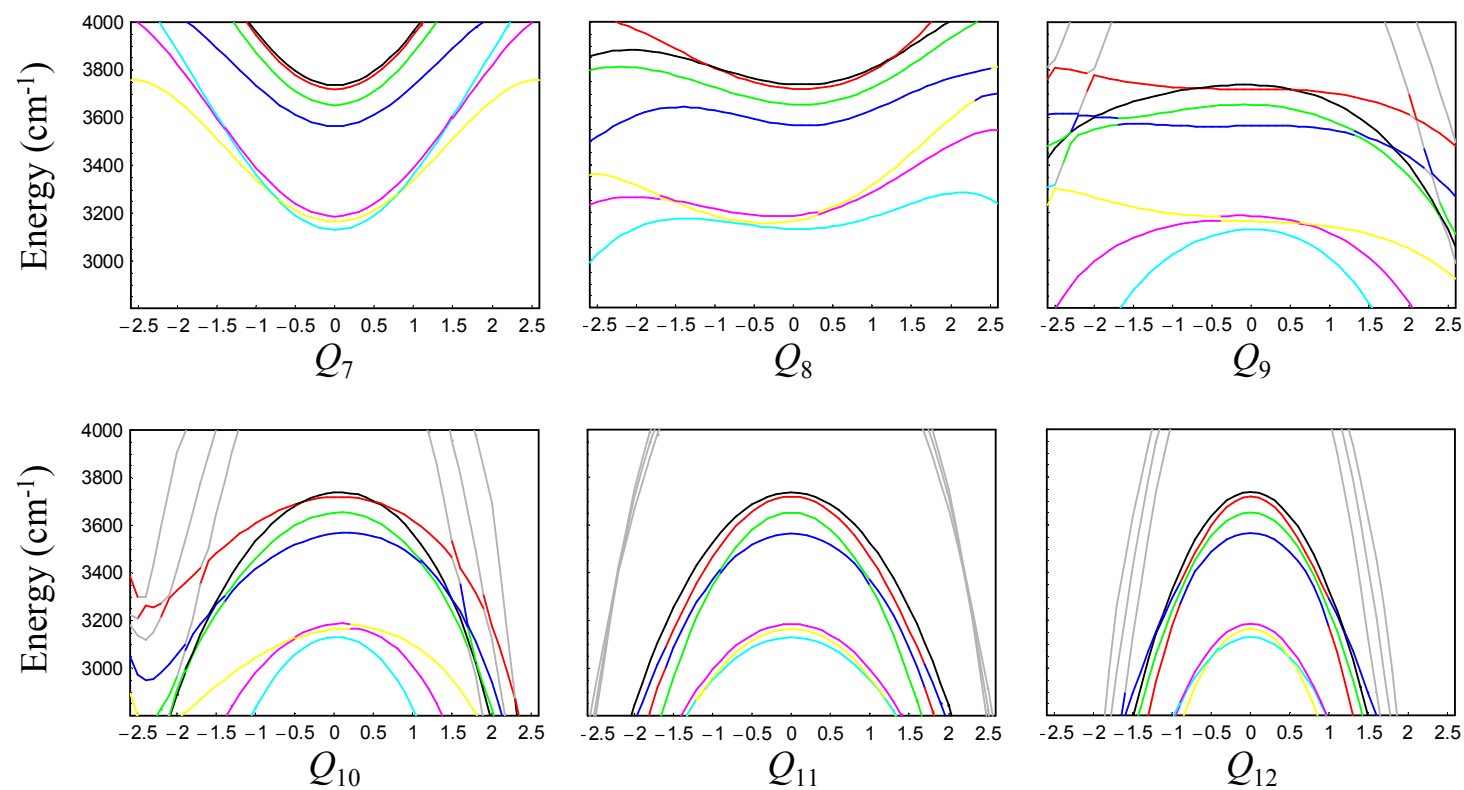

FIG. 4: Normal-mode calculation of the adiabatic PESs, represented by 1D cuts along the lowfrequency normal modes $Q_{7}$ to $Q_{12}$. Shown are all states of the water dimer with either one quantum in the $\mathrm{OH}$ stretch modes or two quanta in the $\mathrm{OH}$ bend modes, that is, $|100000\rangle$

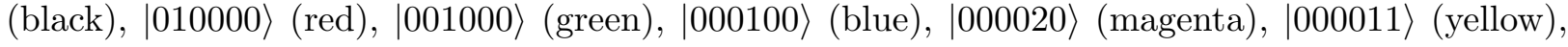
and $|000002\rangle$ (cyan). Curves colored in gray correspond to lower or higher lying states.

Convergence was tested with respect to the number of excitations, as well as the step size and the total size of the $6 \mathrm{D}$ grid.

\section{RESULTS AND DISCUSSION}

\section{A. Normal-mode representation}

In what follows, we focus on the seven states in the $\nu_{1} / \nu_{2}$-diad, i.e., all states with either one quantum in the $\mathrm{OH}$ stretch modes or two quanta in the $\mathrm{OH}$ bend modes. For the lowest energy conformation of the water dimer, the energy ordering of the fully anharmonic states remains the same as for the normal modes in Fig. 1. By sorting the vibrational states from high to low energy, we find: $|100000\rangle$ is the asymmetric OH stretch of the hydrogen-bond accepting water, $|010000\rangle$ the dangling $\mathrm{OH}$ stretch of the hydrogen-bond donating water, $|001000\rangle$ the symmetric $\mathrm{OH}$ stretch of the hydrogen-bond accepting water, $|000100\rangle$ is the stretch of the hydrogen-bonded $\mathrm{H}$ group; $|000020\rangle$ has two quanta of the $\mathrm{OH}$ bend of the hydrogen-bond donating water, $|000002\rangle$ two quanta of the $\mathrm{OH}$ bend of the hydrogen-bond accepting water, and $|000011\rangle$ is a combination mode with one quantum each of the two $\mathrm{OH}$ bends. 
To discuss the adiabatic PESs $W_{k}(Q)$ of these states, we first adopt the the normal-mode representation $\left\{q_{n}, Q_{j}\right\}$ of the parameterized water potential of Bowman [53-55]. Figure 4 shows $1 \mathrm{D}$ cuts of the resulting PESs along the low-frequency modes $\left(Q_{7}, \ldots Q_{12}\right)$. The curves along $Q_{7}$ appear as expected, i.e., (i) they exhibit a minimum at the equilibrium geometry $Q_{7}=0$, (ii) the four states reflecting the $\mathrm{OH}$ stretch excitation are close in energy, as well as (iii) the three states reflecting double excitations of the $\mathrm{OH}$ bends are close. Surprisingly, though, the adiabatic PESs $W_{k}\left(Q_{i}\right)$ along the other normal modes $\left(Q_{8}, \ldots, Q_{12}\right)$ lead mostly 'down-hill', meaning that $W_{k}\left(Q_{i}\right)$ is not minimal at the equilibrium geometry as expected. In fact, these curves would predict a red-shift of the infrared excitation for increasing values of $Q_{i}$, thus rendering the breaking of the hydrogen bond energetically favorably. As this is against common physical intuition, we repeated the calculations using the simple quartic Taylor expansion (Sec. II B) to represent the potential. The results were quite similar and revealed that the negative curvature of the adiabatic PES along the normal modes $Q_{9}$ to $Q_{12}$ is caused by very large negative anharmonic couplings $\propto q_{n}^{2} Q_{j}^{2}$ [cf. Eq. (8)]. For example, the

term that couples modes $q_{3}$ and $Q_{10}$ amounts to $6 g_{33,1010}=-378 \mathrm{~cm}^{-1}$. Together with the also negative zero-point energy contributions of all other high-frequency modes, it accounts for the negative curvature of state $|001000\rangle$ as a function of $Q_{10}$ (green line in Fig. 4) according to Eq. (9). The spurious behavior of the adiabatic PESs shown in Fig. 4 is not an artifact of the electron-structure theory (because we compare the aug-cc-pVTZ/CCSD(T) calculation by Bowman [53-55] with a MP2/6-311++g(2df,2pd) calculation for the Taylor expansion) or a low-order Taylor expansion (because the calculation based on Bowman's potential implicitly includes all terms).

\section{B. Internal mode representation}

Alternatively, we may employ internal modes (Sec. II C) which treat the high-frequency modes in normal mode coordinates that are defined in the frame of the individual water monomers, and thus rotate as the water molecules rotate along the low frequency degrees of freedom (Fig. 2). Proceeding this way, the resulting adiabatic PESs shown in Fig. 5 appear to be quite reasonable, i.e., they exhibit minima very close to the equilibrium geometry $X_{j}=0$.

To facilitate the comparison of the adiabatic PESs calculated in normal modes (Fig. 4) versus those calculated in internal coordinates (Fig. 5), we expand the intermolecular coordinates $\left\{X_{i}\right\}$ in terms of the low-frequency normal modes $\left\{Q_{j}^{\prime}\right\}$ [cf. (Eq. (16)]. This allows us to represent the internal-coordinate PESs in terms of the normal modes $\left\{Q_{j}^{\prime}\right\}$, which is shown in Fig. 6. Again, these adiabatic PESs look very reasonable and clearly confirm the misconception of the PESs calculated directly from normal modes in Fig. 4. 

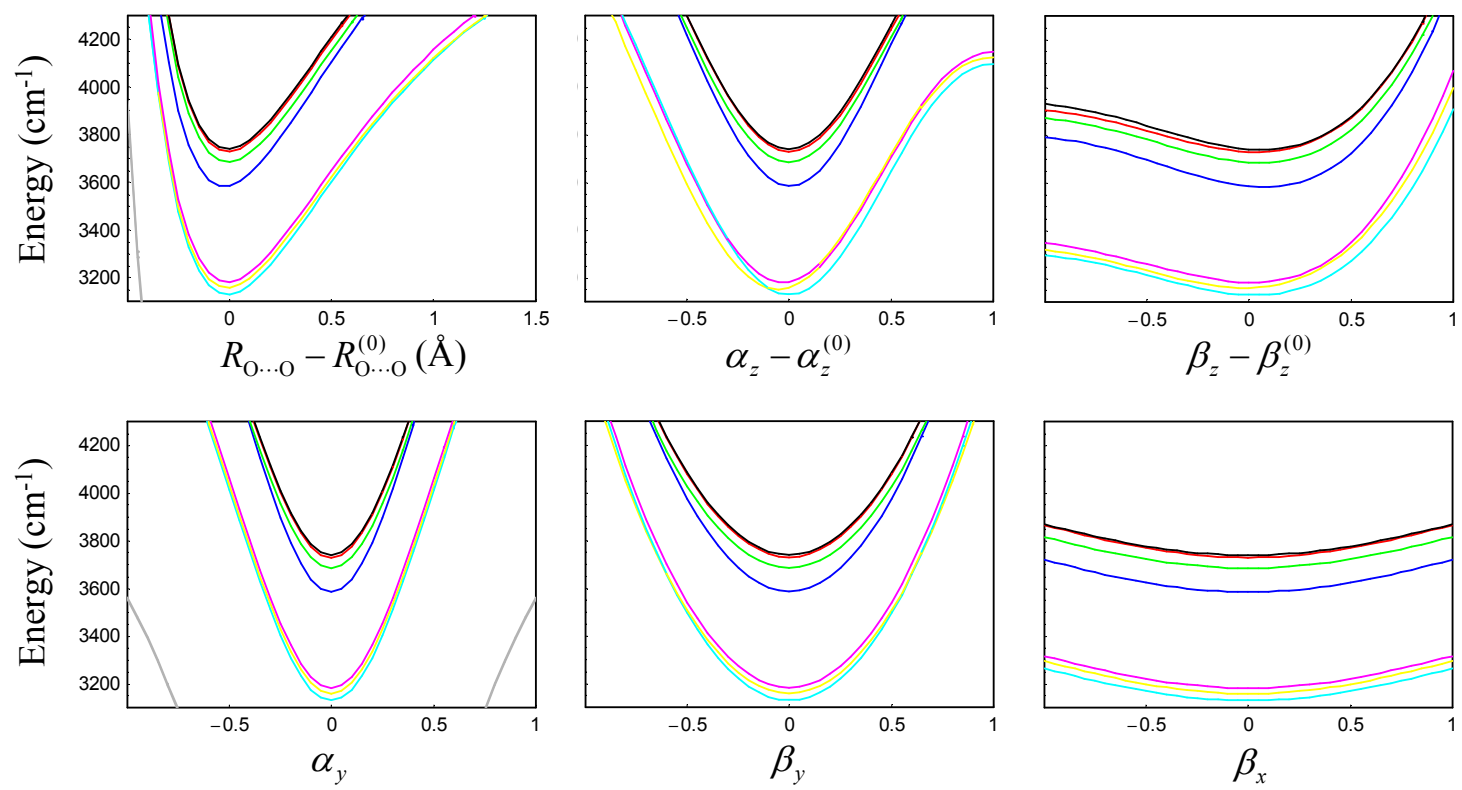

FIG. 5: Internal mode calculation of the adiabatic PESs of the water dimer, using the color code of the states as in Fig. 4. Shown are 1D cuts along the intermolecular coordinates defined in Fig. 2.

\section{Nonadiabatic couplings}

The comparison of Figs. 4 and 6 poses the question of what goes wrong in the normalmode representation. After all, normal modes represent a complete set of coordinates of the system, hence -at least in principle- the problem should be describable also in normal modes. However, one needs to recall that the concept of independent adiabatic PESs rests on the adiabatic approximation, which assumes that (at least for the most part) the nonadiabatic couplings given in Eq. (4) can be neglected. These couplings are given by $\Lambda_{k k^{\prime}}=F_{k k^{\prime}}+G_{k k^{\prime}}$ with

$$
\begin{aligned}
F_{k k^{\prime}} & =-\sum_{j} \frac{\Omega_{j}}{2}\left\langle\phi_{k} \mid \frac{\partial}{\partial Q_{j}} \phi_{k^{\prime}}\right\rangle P_{j}, \\
G_{k k^{\prime}} & =-\sum_{j} \frac{\Omega_{j}}{2}\left\langle\phi_{k} \mid \frac{\partial^{2}}{\partial Q_{j}^{2}} \phi_{k^{\prime}}\right\rangle,
\end{aligned}
$$

and arise from the partitioning in high- and low-frequency modes $\left\{q_{n}\right\}$ and $\left\{Q_{j}\right\}$. Offdiagonal couplings lead to transfer of population between adiabatic states [see Eq. (4)], while diagonal terms may shift the energy of the states and therefore change the appearance of the PESs in Figs. 4 and 6. The diagonal contribution of the first derivative coupling vanishes, $F_{k k}=0$, but the diagonal second derivative coupling is in general non-zero. It can be expressed as [61]

$$
G_{k k}=\sum_{j} \sum_{k \neq k^{\prime}} \frac{\Omega_{j}}{2} \frac{\left\langle\phi_{k^{\prime}}\left|\partial H / \partial Q_{j}\right| \phi_{k}\right\rangle^{2}}{\left(W_{k^{\prime}}-W_{k}\right)^{2}}
$$



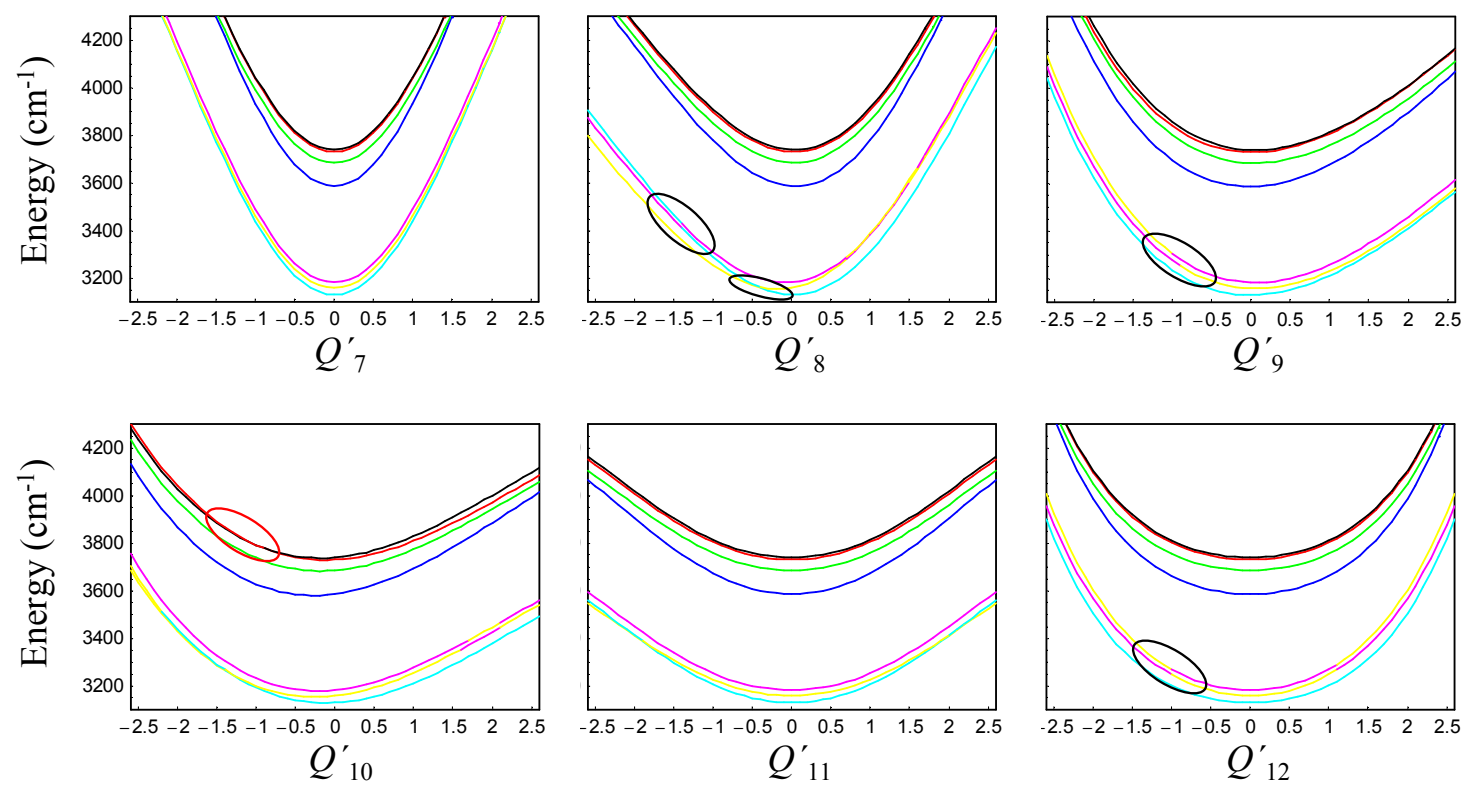

FIG. 6: Internal mode calculation of the adiabatic PESs of the water dimer, using the color code of the states as in Fig. 4. Shown are 1D cuts along the low-frequency normal modes defined in Eq. (16). The encircled regions mark curve crossings; the one in the panel along $Q_{10}^{\prime}$ (encircled in red) is a true crossing of states $|100000\rangle$ (black) and $|010000\rangle$ (red) that leads to the conical intersection shown in Fig. 8.

which is easier to evaluate numerically, and also shows that the diagonal nonadiabatic coupling is always positive. Hence, it increases the energies of the corresponding adiabatic states, which may explain the qualitative difference between Figs. 4 and 6 .

The effect can be illustrated most easily for the vibrational ground state, which is energetically isolated and therefore does not show any curve crossings. Adopting the low-frequency mode $Q_{10}$ as a representative example, Fig. 7 compares the PES (top panels) and the nonadiabatic coupling (bottom panels) as computed in the normal-mode representation (left) and the internal-mode representation (right). The latter shows the familiar adiabatic picture, that is, the PES accounts for the variation of the potential energy along this mode (Fig. 7b), while the nonadiabatic coupling is very small (Fig. 7d). The additional kinetic couplings originating from the curvilinear intermolecular coordinates, not yet included in the derivative couplings Eqs. 19 and 20, are expected to be of the same order of magnitude. In the normal-mode representation, on the other hand, we find a very different situation. Here the diagonal nonadiabatic coupling varies even more strongly with $Q_{10}$ than the corresponding PES (note the very different energy scale in Figs. 7c and 7d, respectively). In other words, the adiabatic approximation breaks down completely. Interestingly, if one adds in the normal-mode representation the energies of the PES and of the diagonal nonadiabatic coupling (i.e., Fig. 7a plus Fig. 7c), the result (Fig. 7b, black line) compares quite well to 

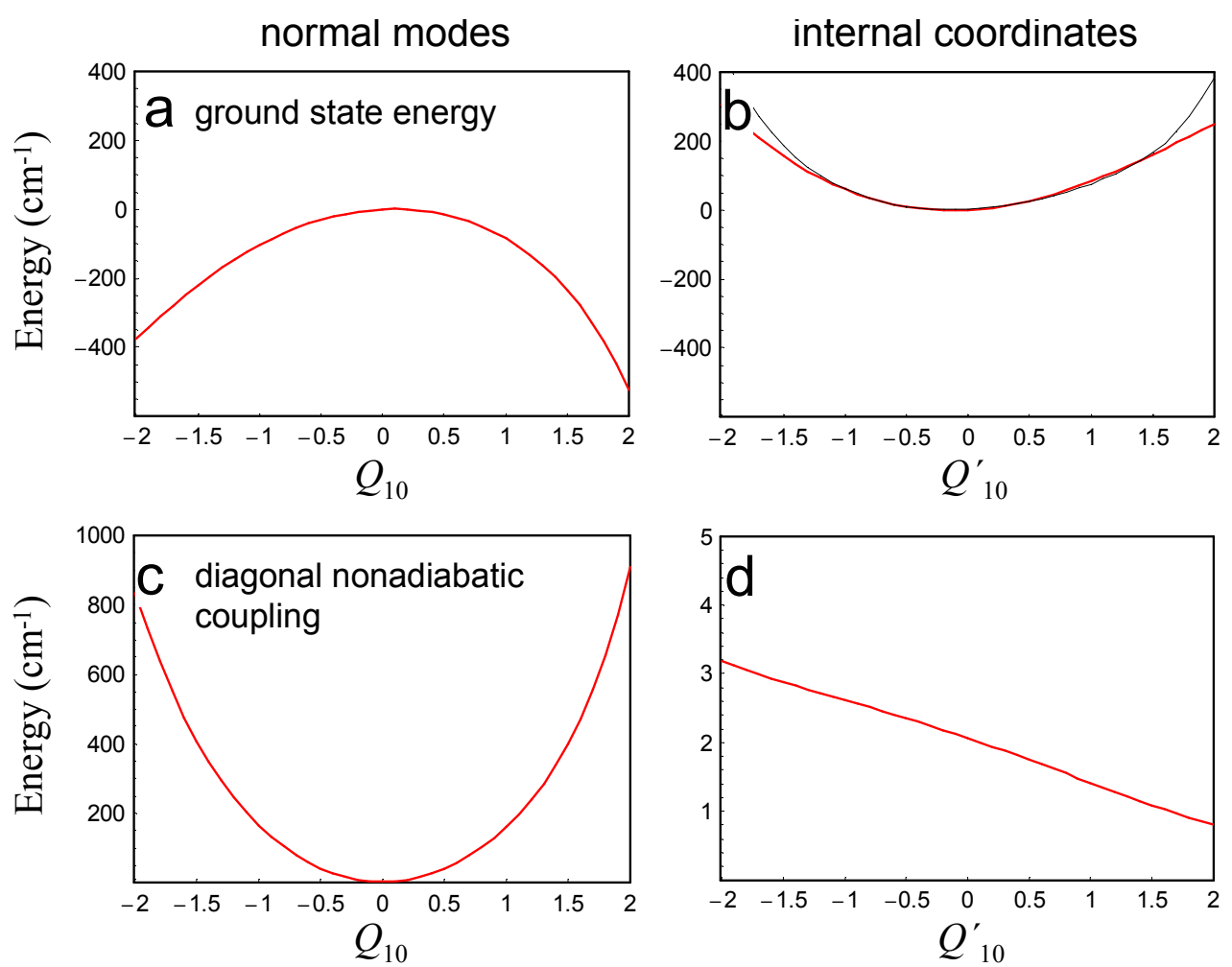

FIG. 7: Break-down of the adiabatic approximation in the normal-mode representation (left panels), illustrated by (a) the ground-state vibrational PES along $Q_{10}$ showing a negative curvature and (c) the corresponding diagonal nonadiabatic coupling which is not only non-zero but dominates the energy. The adiabatic approximation is valid in the internal-mode representation (right panels), where the nonadiabatic coupling almost vanishes (d, note the different energy scale). Panel (b) compares the energy of the ground state PES in internal coordinates (red) with the sum (black) of the PES and the nonadiabatic coupling in the normal-mode representation (i.e., the sum of the energies shown in panels a and c).

the PES in the internal-mode representation (Fig. 7b, red line). The deviation for larger $Q_{10}^{\prime}$ can be explained by the approximation [Eq. (16)] of the curvilinear coordinates $Q_{10}^{\prime}$ by the linear normal coordinates $Q_{10}$. For higher lying states, the picture is similar but in detail more complicated, since curve crossings occur that lead to discontinuities due to the denominator in Eq. (21) and also render off-diagonal couplings more important.

To illustrate the above findings, it is instructive to revisit Fig. 3 which demonstrates the coupling of the high-frequency mode $q_{3}$ to the low-frequency mode $Q_{10}$ in the normal-mode representation. In particular, it reveals that for displaced mode $Q_{10}$, the $\mathrm{OH}$ stretching mode $q_{3}$ no longer displaces the hydrogens parallel to the $\mathrm{OH}$ bonds, but with an angle $\theta$ out of the plane of the hydrogen-bond accepting water. A simple analysis suggests that a term $\propto \cos \theta$ describes the observed negative curvature of the PES as a function of $Q_{10}$. This is because $\cos \theta$ accounts for the projection of $q_{3}$ onto the $\mathrm{OH}$ bond, which corresponds to an intramolecular displacement. In contrast, the component perpendicular to the $\mathrm{OH}$ bond 


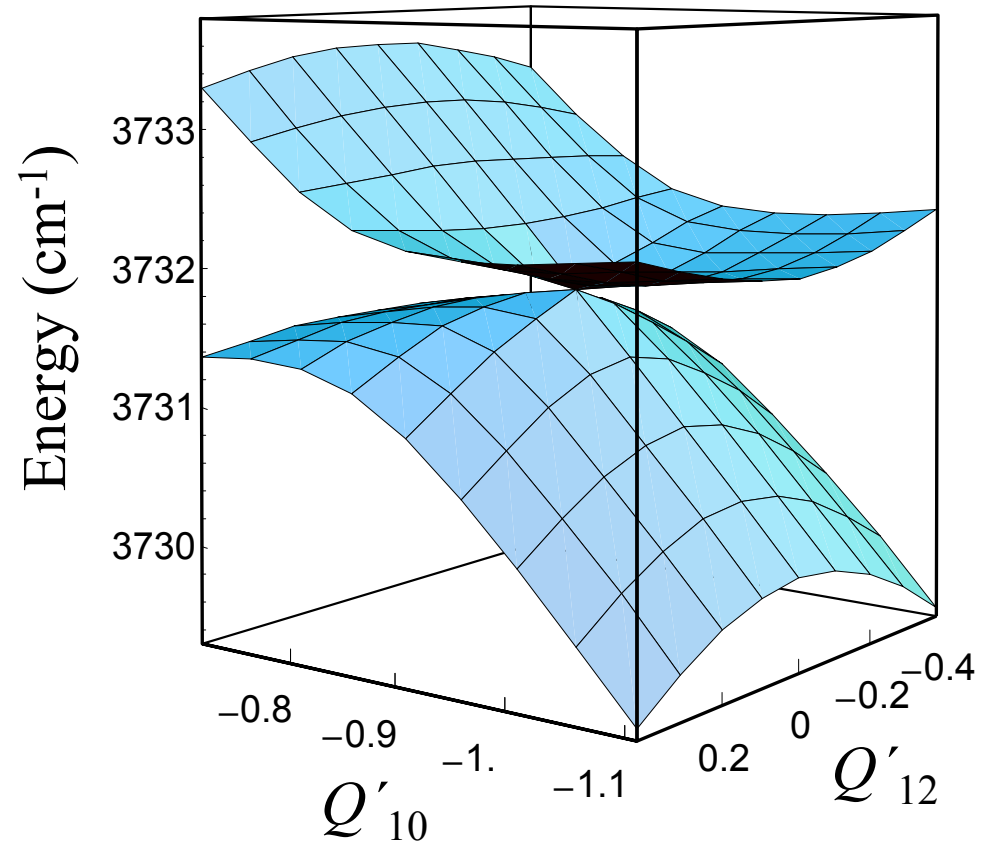

FIG. 8: Conical intersection between states $|100000\rangle\left(A^{\prime \prime}\right)$ and $|010000\rangle\left(A^{\prime}\right)$ as a function of $Q_{10}\left(A^{\prime}\right)$ and $Q_{12}\left(A^{\prime \prime}\right)$. For better visualization, the contribution of the zero-point energy was subtracted.

$(\sin \theta)$ is of intermolecular character and has a much weaker contribution to the potential energy. This mixing of low- and high-frequency degrees of freedom is particularly problematic when we wish to achieve an adiabatic separation, because that separation is no longer well defined when the dimer is not in its lowest energy configuration.

\section{Curve crossings}

Because of the small nonadiabatic couplings, the internal-coordinate PESs (Figs. 6) constitute a physically useful adiabatic picture, and we will focus on them in what follows. It is seen in Fig. 6 that many of the curves are quite close to each other. When we zoom in, we find several crossings of the PESs within the four $\mathrm{OH}$ stretching modes and the three $\mathrm{OH}$ bending modes, respectively, some of which are encircled. While many of these curve crossing are avoided in 1D (the splittings are sometimes hardly visible in Fig. 6), they may lead to 4D seams of conical intersections in full 6D space. Symmetry helps to find such conical intersection in lower-dimensional cuts. For example, a true crossing occurs if a $A^{\prime \prime}$ high-frequency state crosses an $A^{\prime}$ state along a $A^{\prime}$ low frequency tuning mode. Moreover, when we add an additional $A^{\prime \prime}$ coupling mode to that situation in a $2 \mathrm{D}$ cut, this crossing will necessarily become a point-like conical intersection, because the $A^{\prime \prime}$ mode couples both states so the crossing is avoided. 
As a representative example, Fig. 6 shows a curve crossing (encircled in red in Fig. 6) of states $|100000\rangle\left(A^{\prime \prime}\right)$ and $|010000\rangle\left(A^{\prime}\right)$ as a function of normal-mode $Q_{10}\left(A^{\prime}\right)$. It represents a true crossing because both states are of different symmetry ( $A^{\prime \prime}$ vs. $\left.A^{\prime}\right)$. Upon coupling to another $A^{\prime \prime}$ low-frequency mode, such as, e.g., $Q_{12}$, we obtain a conical intersection as shown in Fig. 8.

In contrast to malonaldehyde discussed in Ref. [4], where the hydrogen bond and hence the anharmonic couplings are much stronger, the energy of the conical intersection is not below the Franck-Condon region, such that the crossing may not necessarily play a dominant role in the fast dynamics. Nevertheless, the conical intersection occurs at $Q_{10} \approx-1$, which corresponds to the classical turning point at the zero-point energy, hence the intersection will be seen by any excited wave packet. Accounting for the interaction of the asymmetric $\mathrm{OH}$ stretch vibration of the hydrogen-bond accepting water and the dangling $\mathrm{OH}$ stretch stretch vibration of the hydrogen-bond donating water, this intersection leads to intermolecular vibrational energy transport between the two water molecules.

\section{CONCLUSIONS}

Vibrational conical intersections might provide an efficient mechanism to facilitate the ultrafast vibrational dynamics experimentally found for various forms of water. In a very first step towards this question, we have adopted an adiabatic approach to the vibrational structure of the water dimer, which for long has served as a minimal model for the hydrogen bonding in liquid water. Based on a state-of-the-art potential of $\left(\mathrm{H}_{2} \mathrm{O}\right)_{2}$ and subsequent vibrational-structure calculations, we have calculated the adiabatic PESs for the lowest states of the intramolecular high-frequency modes along the intermolecular low-frequency modes. Although a significant separation of time scales exist between both sets of coordinates, we have found that a standard normal mode description of the adiabatic PESs leads to a qualitatively wrong picture. This complete breakdown of the adiabatic ansatz is caused by strong nonlinear couplings between high- and low-frequency normal modes, which in turn give rise to large overall nonadiabatic couplings. On the other hand, a valid adiabatic picture is obtained, when internal coordinates are employed. Here we have used normal modes to describe the intramolecular motion of each water molecule and intermolecular coordinates to describe the relative motion of the molecules. The chosen coordinates were found to largely decouple the high- and low-frequency degrees of freedom. Of course, the price one has to pay in an internal coordinate representation with curvelinear intermolecular coordinates is a rather complicated kinetic energy operator of the low-frequency degrees of freedom [9] and additional nonadiabatic coupling terms due to kinetic couplings. We however believe that the greatest value of an adiabatic representation lies in the mixed-quantum classical 
treatment of a larger system, e.g., in order to study the vibrational dynamics in bulk water. In this case, that kinetic energy is needed only for the classical sub-system and hence not so difficult to deal with.

Considering 1D and 2D cuts of the adiabatic PESs of $\left(\mathrm{H}_{2} \mathrm{O}\right)_{2}$ along selected intermolecular coordinates, we have been able to show the existence of low-lying vibrational conical intersections in the water dimer. By coupling the motion of the $\mathrm{OH}$ modes, the intersections may mediate the vibrational energy transport between the two water molecules. In a similar vein, recent quantum-classical studies of the vibrational energy transport along the coupled $\mathrm{CO}$ vibrations of a peptide chain have indicated the existence of numerous conical intersections along the classical trajectory [43]. This excitonic-like energy transport along coupled high-frequency oscillators corresponds to almost resonant transitions between almost equivalent system, which means that there is only little excess energy, only little change of the equilibrium geometry, and therefore also only little change of the vibrational dynamics. Although conical intersections have been discussed as a possible mechanism for excitonic energy transport [62], one might expect only minor differences in the effect of true and avoided crossings.

The situation is different if the conical intersection mediates a transition from higher to lower adiabatic PES, thus resulting in the vibrational energy redistribution between, e.g., $\mathrm{OH}$ stretching and $\mathrm{OH}$ bending modes. Due to the typically shifted equilibrium geometry of the lower state and the large excess energy after the transition, the crossing may thoroughly change the vibrational dynamics of the system. In this scenario, a conical intersection that effectively and rapidly transfers population to another state certainly makes a difference to an avoided crossing with significantly less efficient population transfer [63]. In the case of the water dimer, however, we have not found this type of conical intersection. When we extend the investigations to larger water clusters or even bulk water, we will have more $\mathrm{OH}$ modes and thus a higher level density, and would therefore also expect more conical intersections. Unlike to the established role of electronic conical intersections in ultrafast photophysical and photochemical processes, the general importance of vibrational conical intersections to account for ultrafast vibrational dynamics remains yet to be shown.

\section{Acknowledgements}

This paper is dedicated to Martin Quack in honor of his 65th birthday. Furthermore, we thank Joel Bowman for making his potential energy surface of water available and Tucker Carrington for instructive and helpful discussions. The work has been supported in part by 
the Swiss National Science Foundation (SNF) through the NCCR MUST.

[1] J. von Neumann and E. Wigner, Phys. Z. 30, 467 (1929).

[2] W. Domcke, D. R. Yarkony, and H. Köppel, editors, Conical Intersections: Theory, Computation and Experiment, World Scientific, Singapore, 2011.

[3] W. Domcke and D. R. Yarkony, Annu. Rev. Phys. Chem. 63, 325 (2012).

[4] P. Hamm and G. Stock, Phys. Rev. Lett. 109, 173201 (2012).

[5] I. W. M. Smith, Acc. Chem. Res. 23, 101 (1990).

[6] A. Staib and J. T. Hynes, Chem. Phys. Lett. 204, 197 (1993).

[7] W. Klopper, M. Quack, and M. A. Suhm, Chem. Phys. Lett. 261, 35 (1996).

[8] W. Klopper, M. Quack, and M. A. Suhm, J. Chem. Phys. 108, 10096 (1998).

[9] C. Leforestier, F. Gatti, R. S. Fellers, and R. J. Saykally, J. Chem. Phys. 117, 8710 (2002).

[10] K. Giese, M. Petkovic, H. Naundorf, and O. Kühn, Phys. Rep. 430, 211 (2006).

[11] G. L. Barnes, S. M. Squires, and E. L. Sibert, J. Phys. Chem. B 112, 595 (2008).

[12] T. Chiavassa et al., J. Phys. Chem. 96, 10659 (1992).

[13] T. Hayashi and S. Mukamel, J. Phys. Chem. A 107, 9113 (2003).

[14] N. O. B. Lüttschwager, T. N. Wassermann, S. Coussan, and M. A. Suhm, Phys. Chem. Chem. Phys. 12, 8201 (2010).

[15] T. Hammer and U. Manthe, J. Chem. Phys. 134, 224305 (2011).

[16] M. Schröder, F. Gatti, and H.-D. Meyer, J. Chem. Phys. 134, 234307 (2011).

[17] J. Stenger et al., J. Chem. Phys. A 105, 2929 (2001).

[18] D. Madsen et al., Chem. Phys. Lett. 341, 56 (2001).

[19] D. Madsen et al., Bull. Chem. Soc. Jpn. 75, 909 (2002).

[20] K. Heyne, N. Huse, E. T. J. Nibbering, and T. Elsaesser, Chem. Phys. Lett. 369, 591 (2003).

[21] K. Heyne, N. Huse, J. Dreyer, E. T. J. Nibbering, and T. Elsaesser, J. Chem. Phys. 121, 902 (2004).

[22] H. Graener, G. Seifert, and A. Laubereau, Phys. Rev. Lett. 66, 2092 (1991).

[23] S. Woutersen and H. J. Bakker, Nature 402, 507 (1999).

[24] J. Stenger, D. Madsen, P. Hamm, E. T. J. Nibbering, and T. Elsaesser, Phys. Rev. Lett. 87, $027401(2001)$. 
[25] J. B. Asbury et al., J. Chem. Phys. 121, 12431 (2004).

[26] S. Yeremenko, M. S. Pshenichnikov, and D. A. Wiersma, Chem. Phys. Lett. 369, 107 (2003).

[27] J. D. Eaves et al., Proc. Natl. Acad. Sci. USA 102, 13019 (2005).

[28] A. M. Dokter and H. J. Bakker, J. Chem. Phys. 128, 024502 (2008).

[29] H. J. Bakker and J. L. Skinner, Chem. Rev. 110, 1498 (2010).

[30] H.-K. Nienhuys, S. Woutersen, R. A. van Santen, and H. J. Bakker, J. Chem. Phys. 111, 1494 (1999).

[31] J. C. Deàk, S. T. Rhea, L. K. Iwaki, and D. D. Dlott, J. Phys. Chem. A 104, 4866 (2000).

[32] M. L. Cowan et al., Nature 434, 199 (2005).

[33] L. Piatkowski, K. B. Eisenthal, and H. J. Bakker, Phys. Chem. Chem. Phys. 11, 9033 (2009).

[34] F. Perakis and P. Hamm, Phys. Chem. Chem. Phys. 14, 6243 (2012).

[35] A. Laubereau and W. Kaiser, Rev. Mod. Phys. 50, 607 (1978).

[36] J. C. Owrutsky, D. Raftery, and R. M. Hochstrasser, Annu. Rev. Phys. Chem. 45, 519 (1994).

[37] R. Rey, K. B. Moller, and J. T. Hynes, Chem. Rev. 104, 1915 (2004).

[38] H. Fujisaki, Y. Zhang, and J. E. Straub, Adv. Chem. Phys. 145, 1 (2011).

[39] M. Yang and J. L. Skinner, Phys. Chem. Chem. Phys. 12, 982 (2010).

[40] F. Li and J. L. Skinner, J. Chem. Phys. 133, 244504 (2010).

[41] A. Paarmann, T. Hayashi, S. Mukamel, and R. J. D. Miller, J. Chem. Phys. 130, 204110 (2009).

[42] T. la Cour Jansen and J. Knoester, J. Phys. Chem. B 110, 22910 (2006).

[43] M. Kobus, R. D. Gorbunov, P. H. Nguyen, and G. Stock, Chem. Phys. 347, 208 (2008).

[44] Z. S. Huang and R. E. Miller, J. Chem. Phys 91, 6613 (1989).

[45] S. S. Xantheas and T. Dunning, J. Chem. Phys. 99, 8774 (1993).

[46] S. Scheiner, Annu. Rev. Phys. Chem. 45, 23 (1994).

[47] H. Chen, S. Liu, and J. C. Light, J. Chem. Phys. 110, 168 (1999).

[48] R. S. Fellers, C. Leforestier, L. B. Braly, M. G. Brown, and R. J. Saykally, Science 284, 945 (1999).

[49] G. S. Tschumper et al., J. Chem. Phys. 116, 690 (2002).

[50] N. Goldman et al., J. Chem. Phys. 116, 10148 (2002).

[51] R. Kumar, F.-F. Wang, G. R. Jenness, and K. D. Jordan, J. Chem. Phys. 132, 014309 (2010).

[52] K. Kuyanov-Prozument, M. Y. Choi, and A. F. Vilesov, J. Chem. Phys. 132, 014304 (2010). 
[53] X. Huang, B. J. Braams, and J. M. Bowman, J. Phys. Chem. A 110, 445 (2006).

[54] Y. Wang, B. C. Shepler, B. J. Braams, and J. M. Bowman, J. Chem. Phys. 131, 054511 (2009).

[55] Y. Wang, B. C. Shepler, B. J. Braams, and J. M. Bowman, J. Chem. Phys. 134, 094509 (2011).

[56] H. Köppel, W. Domcke, and L. S. Cederbaum, Adv. Chem. Phys. 57, 59 (1984).

[57] W. H. Miller, N. C. Handy, and J. E. Adams, J. Chem. Phys. 72, 99 (1980).

[58] A. Hoy, I. Mills, and G. Strey, Mol. Phys. 24, 1265 (1972).

[59] M. J. Frisch et al., Gaussian 09 Revision A.1, Gaussian Inc. Wallingford CT, 2009.

[60] D. T. Colbert and W. H. Miller, J. Chem. Phys 96, 1982 (1992).

[61] J. Morelli and S. Hammes-Schiffer, Chem. Phys. Lett. 269, 161 (1997).

[62] S. Wüster, A. Eisfeld, and J. M. Rost, Phys. Rev. Lett. 106, 153002 (2011).

[63] B. Balzer, S. Hahn, and G. Stock, Chem. Phys. Lett. 379, 351 (2003). 